\title{
Insegurança alimentar em um município do extremo sul do Rio Grande do Sul, 2016: um estudo de base populacional* \\ doi: $10.5123 /$ S1679-49742020000200007
}

\author{
Food insecurity in a city in the extreme south of Rio Grande do Sul, Brazil, 2016: a \\ population-based study
}

\section{Inseguridad alimentaria en un municipio del extremo sur de Rio Grande do Sul, Brasil, 2016: un estudio de base poblacional}

\author{
Mariane da Silva Dias ${ }^{1}$ - (1) orcid.org/0000-0003-4995-4748 \\ Samuel de Carvalho Dumith ${ }^{1}$ - (1) orcid.org/0000-0002-5994-735X \\ Juliana dos Santos Vaz ${ }^{2}$ - (1) orcid.org/0000-0002-2880-767X \\ Lulie Rosane Odeh Susin ${ }^{1}$ - (1) orcid.org/0000-0002-9404-0577 \\ 'Universidade Federal do Rio Grande, Faculdade de Medicina, Rio Grande, RS, Brasil \\ Universidade Federal de Pelotas, Faculdade de Nutrição, Pelotas, RS, Brasil
}

\section{Resumo}

objetivo: analisar a ocorrência de insegurança alimentar e fatores associados em Rio Grande, RS, Brasil, em 2016. Métodos: estudo transversal de base populacional, cujos dados foram coletados mediante entrevista com os responsáveis pelos domicílios; empregou-se regressão de Poisson, com ajuste robusto da variância. Resultados: foram incluídos 675 domicílios; a prevalência de insegurança alimentar foi de $35,2 \%\left(\mathrm{IC}_{950} 31,6 ; 39,0\right)$ e sua ocorrência esteve associada ao responsável pelo domicílio ser do sexo feminino $\left(\mathrm{RP}=1,49-\mathrm{IC}_{95 \%} 1,17 ; 1,90\right)$, de raça/cor da pele não branca ( $\left.\mathrm{RP}=1,49-\mathrm{IC}_{95 \%} 1,18 ; 1,88\right)$, apresentar menor faixa etária, não ser casado $\left(\mathrm{RP}=1,39-\mathrm{IC}_{95 \%} 1,07 ; 1,81\right)$, pertencer ao grupo de menor escolaridade $\left(\mathrm{RP}=1,58-\mathrm{IC}_{95 \%} 1,17 ; 2,12\right)$, encontrar-se no primeiro ou segundo tercil de índice de bens, não ter dinheiro suficiente para as despesas $\left(\mathrm{RP}=2,22-\mathrm{IC}_{95 \%} 1,76 ; 2,80\right)$, ser obeso $(\mathrm{RP}=1,39$ $\left.\mathrm{IC}_{95 \%} 1,13 ; 1,71\right)$ e ser fumante $\left(\mathrm{RP}=1,28-\mathrm{IC}_{95 \%} 1,05 ; 1,56\right)$. Conclusão: a insegurança alimentar esteve associada a todos os fatores estudados, exceto consumo abusivo de álcool.

Palavras-chaves: Segurança Alimentar e Nutricional; Fatores Socioeconômicos; Vulnerabilidade Social; Inquéritos Epidemiológicos; Estudos Transversais.

\footnotetext{
*Artigo derivado da dissertação de mestrado intitulada 'Insegurança alimentar no extremo sul do Brasil: um estudo de base populacional', defendida por Mariane da Silva Dias junto ao Programa de Pós-Graduação em Saúde Pública, da Universidade Federal do Rio Grande, em 2017. O presente estudo faz parte de um consórcio de pesquisa intitulado 'Saúde da População Riograndina', financiado com recursos do Programa de Pós-Graduação em Saúde Pública (PPGSP) e do Programa de Pós-Graduação em Ciências da Saúde (PPGCS), ambos vinculados à Faculdade de Medicina da Universidade Federal do Rio Grande, e recebeu apoio financeiro da Fundação de Amparo à Pesquisa do Estado do Rio Grande do Sul (FAPERGS): Programa Primeiros Projetos, ARD/PPP 2014 Processo no 16/2551-0000359-9. Samuel de Carvalho Dumith é bolsista de produtividade em pesquisa do Conselho Nacional de Desenvolvimento Cientifico e Tecnológico/Ministério da Ciência, Tecnologia, Inovações e Comunicações (CNPq/MCTIC): Processo n 306964/2016-3. A Mariane da Silva Dias foi concedida bolsa de mestrado pela Coordenação de Aperfeiçoamento de Pessoal de Nível Superior/Ministério da Educação (Capes/MEC): código do financiamento 001.
} 


\section{Introdução}

A segurança alimentar e nutricional consiste na garantia do acesso regular e permanente a alimentos de qualidade e quantidade suficientes, sem comprometer 0 acesso às demais necessidades essenciais. ${ }^{1}$ Para tanto, o poder público deve adotar políticas e ações que levem em conta fatores ambientais, culturais, econômicos, regionais e sociais, com o objetivo de assegurar esse direito aos indivíduos. ${ }^{2}$

Apesar de a alimentação ser considerada um direito fundamental, a despeito da fome ser reconhecida como o maior problema solucionável para a humanidade, 0 número de pessoas com fome no mundo continua elevado. ${ }^{3,4} 0$ Brasil alcançou destaque positivo na redução da pobreza extrema e da fome, mediante a implementação de políticas bem-sucedidas de inclusão social e programas de transferência de renda desde os anos 2000. ${ }^{5}$ Não obstante esses avanços, a insegurança alimentar (IA) ainda é uma questão presente e importante no país. ${ }^{5}$

As prevalências de IA mostram grande variação, decorrente das diferenças sociais e econômicas entre as grandes regiões nacionais. ${ }^{6,7}$ Segundo dados da Pesquisa Nacional por Amostra de Domicílios (PNAD), realizada pelo Instituto Brasileiro de Geografia e Estatística (IBGE), no ano de 2013, a ocorrência de IA variou entre mais de $36,0 \%$ nas regiões Norte e Nordeste a menos de 15,0\% nas regiões Sul e Sudeste. ${ }^{8}$

\section{A segurança alimentar e nutricional consiste na garantia do acesso regular e permanente a alimentos de qualidade e quantidade suficientes, sem comprometer o acesso às demais necessidades essenciais}

Estudo realizado sobre dados da PNAD, com a finalidade de avaliar tendência e fatores associados à IA no Brasil, encontrou redução em sua prevalência no período 2004-2013, principalmente quanto à IA moderada e grave, de $17,0 \%\left(\mathrm{IC}_{95 \%} 15,7 ; 18,4\right)$ para $7,9 \%$ $\left(\mathrm{IC}_{95 \%} 7,2 ; 8,7\right)$. Porém, observou-se que tal redução foi maior nos municípios que apresentavam menor prevalência em 2004, mostrando a permanência das disparidades relacionadas à IA. ${ }^{7}$ Outro estudo, também com dados da PNAD, gerou estimativa de prevalência de IA moderada ou grave para os municípios brasileiros, destacando sua grande heterogeneidade, especialmente a significativa diferença entre a média dos municípios das regiões Nordeste $(14,5 \%)$ e Sul $(3,6 \%){ }^{6}$

0 monitoramento do estado de segurança alimentar vem sendo realizado no Brasil desde 2004, pela mesma PNAD, com o propósito de classificar os domicílios de acordo com a situação da segurança alimentar e produzir informações básicas sobre o desenvolvimento socioeconômico do país. ${ }^{8}$ Entretanto, as informações geradas pela PNAD podem não retratar a realidade de um município ou microrregião, visto que são apresentados resultados médios de IA para uma região definida. 0 Brasil apresenta grande diversidade inter e intrarregional, motivo pelo qual se faz necessário o monitoramento específico de IA nos municípios ou microrregiões para subsidiar políticas públicas mais adequadas aos respectivos contextos.

Ademais, a IA é um fenômeno complexo, difícil de mensurar apenas com base na Escala Brasileira de Insegurança Alimentar (EBIA), instrumento incapaz de revelar um panorama mais amplo das condições que levam à IA ou que contribuem, conjuntamente, para ela. ${ }^{9}$ Deve-se aplicar, juntamente com a EBIA, outros instrumentos que permitam classificar os domicílios e/ou seus moradores de acordo com indicadores sociais. Estes indicadores possibilitam a identificação dos domicílios com maior nível de vulnerabilidade social. ${ }^{10,11}$

Apesar da importância da obtenção de dados referentes à insegurança alimentar por regiões, no planejamento e implementação de políticas públicas de acesso aos alimentos em quantidade suficiente e necessária, há poucos estudos com dados sobre IA em municípios da zona sul de Rio Grande do Sul (RS). Por exemplo, na cidade de Rio Grande, RS, que nos últimos anos passou por um período de grande instabilidade econômica, quando a taxa de desemprego aumentou muito em decorrência de demissões relacionadas à produção de plataformas no Polo Naval, a maior instabilidade financeira da população acarreta maior incerteza e dificuldade de acesso aos alimentos, causadas pela queda na renda familiar. ${ }^{12}$

0 objetivo do presente artigo foi analisar a ocorrência de insegurança alimentar e fatores associados, no município de Rio Grande, RS, Brasil.

\section{Métodos}

Estudo transversal de base populacional, realizado no município de Rio Grande. Localizado no litoral sul do Rio Grande do Sul, a 317 quilômetros da capital Porto Alegre, Rio Grande possui uma população de 207.036 
habitantes e 66.554 domicílios em sua zona urbana. ${ }^{13}$ A economia local se concentra, em sua maior parte, na atividade portuária. ${ }^{6}$

Este estudo faz parte de um consórcio de pesquisa intitulado 'Saúde da População Riograndina', cujo objetivo foi avaliar diversos desfechos em saúde na população de 18 anos ou mais de idade, residente na cidade no ano de 2016. Mais informações sobre os métodos adotados estão disponíveis em artigo metodológico. ${ }^{14}$

Foram elegíveis para o estudo indivíduos com idade igual ou superior a 18 anos, residentes na zona urbana de Rio Grande. Não foram considerados elegíveis os indivíduos institucionalizados em asilos, hospitais e presídios, e aqueles com incapacidade física e/ou cognitiva para responder ao questionário proposto pelos pesquisadores.

No cálculo do tamanho amostral, foi utilizada uma prevalência do desfecho de $14,9 \%,{ }^{6}$ nível de confiança de $95 \%$, poder estatístico de $80 \%$ e margem de erro de 3,0 pontos percentuais, gerando-se uma amostra de 541 domicílios, sobre a qual foi calculado um efeito de delineamento (def) de 1,3, obtendo-se uma amostra de 703 domicílios, à qual se somaram 10\% para possíveis perdas e recusas. Desta maneira, uma amostra preliminar consistiu de 773 domicílios.

0 processo de amostragem ocorreu em dois estágios. Primeiramente, foram selecionados os setores censitários e, em seguida, os domicílios e os indivíduos. Assim, foram selecionados, de forma sistemática, 72 setores censitários - entre os 293 elegíveis, representando $25 \%$ dos mesmos. Isso resultaria em uma média de dez domicílios por setor. Ao final desse processo, foram selecionados 711 domicílios.

A unidade de análise do estudo foi o domicílio. As perguntas relacionadas ao domicílio e ao desfecho 'insegurança alimentar' foram aplicadas somente ao indivíduo considerado pelos demais moradores como responsável pelo domicílio. Tendo-se como referência a classificação adotada pelo IBGE, ${ }^{14} 0$ estudo considerou como responsável aquele que conhecesse a dinâmica alimentar e financeira do domicílio, ou seja, o indivíduo envolvido na aquisição dos gêneros alimentícios para o consumo da família.

A medição da IA utilizou-se da EBIA. ${ }^{6}$ Cumpre mencionar que se trata de um instrumento adaptado daquele proposto pelo United States Department of Agriculture (USDA). ${ }^{1}$ A EBIA mensura a percepção das famílias sobre seu acesso aos alimentos nos últimos 90 dias que antecederam à entrevista, tendo sido adaptada e validada para melhor corresponder às características da população brasileira. ${ }^{15}$

A IA pode ser leve, moderada ou grave, conforme a somatória das respostas afirmativas da EBIA. Um domicílio é classificado com segurança alimentar quando tem pontuação igual a zero na escala, significando que o domicílio tem acesso a alimentos em quantidade e qualidade suficientes para 0 consumo da família. 0 domicílio é classificado com IA leve quando sua pontuação na escala é de 1 a 3, IA moderada quando pontua de 4 a 5, e IA grave quando sua pontuação se encontra entre 6 e 8 na EBIA. ${ }^{6}$ Foram mantidas as quatro categorias de IA, para se verificar a prevalência de cada uma delas. Nas análises de fatores associados, a variável IA foi utilizada de forma dicotômica: sim ou não.

As variáveis independentes adotadas foram:

a) sexo (masculino; feminino);

b) raça/cor da pele (branca; não branca);

c) faixa etária (em anos: 18-39; 40-59; 60 ou mais);

d) escolaridade (em anos de estudo: 0-8; 9-11; 12 ou mais);

e) índice de bens (categorizado em tercis);

f) número de moradores no domicílio (até 3; 4 ou mais);

g) dinheiro suficiente para pagar as despesas (não; sim);

h) estado conjugal (casado; solteiro/viúvo/separado);

i) tabagismo atual (não; sim);

j) consumo abusivo de álcool (não; sim); e

k) estado nutricional (baixo peso; eutrófico; sobrepeso; obeso). ${ }^{16}$

A variável 'índices de bens' foi gerada para ser uma variável proxy de renda familiar, visto que a variável 'renda' pode ser de pouca confiabilidade: existe a possibilidade de o responsável pelo domicílio se recusar a responder sobre sua renda ou mesmo ignorar o rendimento dos demais moradores do domicílio. ${ }^{17,18}$ Sendo assim, a variável 'índice de bens' foi construída por meio de análise de componentes principais, a partir de 11 variáveis (origem da água utilizada para beber, número de cômodos do domicílio usados para dormir, número de banheiros, posse de carro, posse de computador, acesso a internet, existência de telefone fixo, presença de micro-ondas, máquina de lavar roupa, máquina de secar roupa, e DVD). 0 escore foi dividido em tercis, sendo o primeiro o de pior índice de bens e 0 terceiro o de melhor índice de bens. Foi extraído o primeiro componente que apresentou eingenvalue de 3,3, explicando 30\% da variabilidade de todos os indicadores; estes apresentaram coeficiente de covariância acima de 0,20. 
0 estado nutricional foi caracterizado a partir das informações autorreferidas de peso atual e altura, aplicando-se a fórmula do índice de massa corporal (IMC: $\mathrm{kg} / \mathrm{m}^{2}$ ); ele foi classificado de acordo com os pontos de corte estabelecidos pela Organização Mundial da Saúde (OMS).

0 consumo abusivo de álcool foi definido da seguinte forma: ingestão de cinco ou mais doses de bebida alcoólica para homens e quatro ou mais doses para mulheres, em uma única ocasião nos últimos 30 dias anteriores à entrevista. ${ }^{19}$

0 controle de qualidade dos dados foi efetuado mediante uma segunda entrevista realizada em 10\% dos domicílios visitados, selecionados ao acaso. Onze perguntas-chave foram reaplicadas, sobre idade, raça/ cor da pele, status de fumo no domicílio, existência de telefone fixo, hipertensão, asma, vacina da gripe, se havia moradores menores de 18 anos de idade, número de dias de caminhada/semana, se gosta de morar no bairro e frequência da escovação dos dentes. Essa segunda entrevista foi realizada por telefone e a concordância entre a primeira e a segunda foi testada pelo índice Kappa, cujo valor médio de 0,80 mostrou excelente consistência dos dados.

Os dados foram analisados utilizando-se programa Stata versão 14.1 (Stata Corp. College Station, Estados Unidos). As análises foram realizadas pelo módulo svy, que leva em consideração o desenho amostral. Inicialmente foi realizada uma descrição da amostra em percentuais e números absolutos; e posteriormente, uma análise multivariável utilizando-se a regressão de Poisson, com ajuste robusto para variância.

Foram realizadas análises relacionadas ao domicílio, quando foram incluídas variáveis sociodemográficas, bem como as referentes ao indivíduo responsável - variáveis comportamentais e de estado nutricional.

As análises relacionadas ao domicílio seguiram a ordem do modelo de análise: no primeiro nível, variáveis demográficas (sexo, raça/cor da pele, idade e estado conjugal); no segundo nível, variáveis socioeconômicas (escolaridade, número de moradores no domicílio, índice de bens e dinheiro suficiente); e no terceiro nível, as variáveis comportamentais (estado nutricional, tabagismo atual e consumo abusivo de álcool).

Tal estratégia de análise pressupõe a inclusão no modelo de ajuste de todas as variáveis sob estudo, com método de inclusão backward segundo o modelo de análise previamente determinado. Em cada nível, foi realizado o ajuste e identificadas as variáveis com $\mathrm{p}>0,20$; estas foram excluídas do modelo e não entraram no ajuste dos níveis seguintes. Essa estratégia de análise e a manutenção no modelo de variáveis com valor de $\mathrm{p}<0,20$ têm sido recomendadas, para melhor ajuste de fatores de confusão. 0 nível de significância foi estimado utilizando-se o teste de Wald para heterogeneidade; as associações com valor de $\mathrm{p} \leq 0,05$ foram consideradas estatisticamente significativas.

0 projeto da pesquisa foi aprovada pelo Comitê de Ética de Pesquisa na área da Saúde da Universidade Federal do Rio Grande (FURG), conforme Ofício $n^{0}$ 20/2016, de março de 2016; o Termo de Consentimento Livre e Esclarecido foi obtido de todos os entrevistado s.

\section{Resultados}

Ao final, a amostra constituiu-se de 675 domicílios, perdas e recusas totalizaram $5,1 \%$. As perdas foram maiores em setores censitários do centro da cidade de Rio Grande (20,0\%), bem como entre homens (12,0\% versus $6,5 \%$ para mulheres). Com relação aos indivíduos responsáveis pelo domicílio, 61,6\% eram do sexo feminino, $83,2 \%$ autodeclararam-se de raça/cor da pele branca e 41,2\% encontravam-se na faixa etária de 40 a 59 anos (Tabela 1).

A ocorrência de IA nos domicílios de Rio Grande foi de $35,2 \%$ ( IC $_{95 \%} 31,6 \% ; 39,0 \%$ ), sendo $26,3 \%$ de IA leve (IC $\left.{ }_{95 \%} 29,8 \% ; 23,0 \%\right), 6,1 \%$ de IA moderada $\left(\mathrm{IC}_{95 \%} 8,2 \% ; 4,4 \%\right)$ e 2,8\% de IA grave ( $\left.\mathrm{IC}_{95 \%} 4,4 \% ; 1,7 \%\right)$.

A Tabela 2 apresenta as medidas de associação brutas e ajustadas, entre as variáveis e o desfecho IA. Após o ajuste, todas as variáveis, exceto o consumo abusivo de álcool, apresentaram associação estatisticamente significativa com o desfecho. Domicílios cuja responsável era do sexo feminino apresentaram prevalência de IA 49\% maior, em comparação àqueles cujo responsável era do sexo masculino ( $\left.\mathrm{RP}=1,49-\mathrm{IC}_{95 \%} 1,17 ; 1,90\right)$, assim como aqueles cujo responsável se declarou de raça/cor da pele não branca, em relação àqueles chefiados por pessoa de raça/cor da pele branca $\left(\mathrm{RP}=1,49 ; \mathrm{IC}_{95 \%} 1,18 ; 1,88\right)$.

Domicílios chefiados por pessoas com idades de 18 a 39 anos e 40 a 59 anos apresentaram maior probabilidade de IA quando comparados àqueles cujos chefes pertenciam à faixa etária de 60 anos ou mais $\left(\mathrm{RP}=1,93-\mathrm{IC}_{95 \%} 1,42 ; 2,62\right.$ e $\mathrm{RP}=1,38-\mathrm{IC}_{95 \%} 1,33 ; 2,44$, respectivamente). Observou-se, portanto, uma tendência linear segundo a qual, conforme aumentava a faixa 
Tabela 1 - Descrição da amostra de acordo com características socioeconômicas, demográficas, comportamentais e estado nutricional ( $n=675$ ), Rio Grande, $R S, 2016$

\begin{tabular}{|c|c|c|}
\hline Variáveis & $\mathbf{n}$ & $\%$ \\
\hline \multicolumn{3}{|l|}{ Sexo } \\
\hline Masculino & 259 & 38,4 \\
\hline Feminino & 416 & 61,6 \\
\hline \multicolumn{3}{|l|}{ Raça/cor da pele ${ }^{a}$} \\
\hline Branca & 561 & 83,2 \\
\hline Preta, parda e outras & 113 & 16,8 \\
\hline \multicolumn{3}{|l|}{ Faixa etária (em anos) } \\
\hline $18-39$ & 191 & 28,3 \\
\hline $40-59$ & 278 & 41,2 \\
\hline$\geq 60$ & 206 & 30,5 \\
\hline \multicolumn{3}{|c|}{ Escolaridade (em anos de estudo) ${ }^{a}$} \\
\hline $0-8$ & 301 & 44,6 \\
\hline $9-11$ & 196 & 29,1 \\
\hline$\geq 12$ & 177 & 26,3 \\
\hline \multicolumn{3}{|c|}{ Número de moradores no domicílio } \\
\hline Até 3 & 487 & 72,2 \\
\hline 4 ou mais & 188 & 27,8 \\
\hline \multicolumn{3}{|l|}{ Índice de bens } \\
\hline $1^{0}$ (pior situação) & 273 & 40,5 \\
\hline $2^{0}$ & 208 & 30,8 \\
\hline $3^{\circ}$ (melhor situação) & 194 & 28,7 \\
\hline \multicolumn{3}{|c|}{ Dinheiro suficiente para pagar as despesas ${ }^{b}$} \\
\hline Não/em parte & 258 & 38,4 \\
\hline Sim & 414 & 61,6 \\
\hline \multicolumn{3}{|l|}{ Estado conjugal } \\
\hline Casado & 247 & 36,6 \\
\hline Solteiro & 264 & 39,1 \\
\hline Viúvo/separado & 164 & 24,3 \\
\hline \multicolumn{3}{|l|}{ Estado nutricionalc } \\
\hline Baixo peso & 11 & 1,7 \\
\hline Eutrófico & 246 & 37,9 \\
\hline Sobrepeso & 244 & 37,7 \\
\hline Obeso & 147 & 22,7 \\
\hline \multicolumn{3}{|l|}{ Tabagismo atual } \\
\hline Não fumante & 533 & 79,0 \\
\hline Fumante & 142 & 21,0 \\
\hline \multicolumn{3}{|c|}{ Consumo abusivo de álcool ${ }^{a}$} \\
\hline Não & 599 & 88,9 \\
\hline Sim & 75 & 11,1 \\
\hline
\end{tabular}

a) 1 missing na variável.

b) 3 missings na variável.

c) 27 missings na variável.

etária, reduzia-se a probabilidade de apresentar IA. A prevalência de IA foi superior nos domicílios cujos responsáveis não eram casados $\left(\mathrm{RP}=1,39-\mathrm{IC}_{95 \%} 1,07 ; 1,81\right)$, relativamente aos casados.

Encontrou-se maior probabilidade de IA nos domicílios cujos responsáveis tinham até oito anos de estudo $\left(\mathrm{RP}=1,58-\mathrm{IC}_{95 \%} 1,17 ; 2,12\right)$, comparados aos que tinham 12 ou mais anos de vida escolar. Da mesma forma, pertencer aos terços de bens inferiores esteve associado à maior probabilidade de apresentar IA $(\mathrm{RP}=2,40-$ $\mathrm{IC}_{95 \%} 1,66 ; 3,46\left[1^{\circ}\right.$ terço] e $\mathrm{RP}=2,13-\mathrm{IC}_{95 \%} 1,49 ; 3,05\left[2^{\circ}\right.$ terço]), na comparação com pertencer ao terço superior. Domicílios com quatro ou mais moradores revelaram maior probabilidade de apresentar IA $(\mathrm{RP}=1,32$ - $\left.\mathrm{IC}_{95 \%} 1,12 ; 1,56\right)$, quando comparados aos domicílios com menos moradores. Domicílios cujos responsáveis declararam não ter dinheiro suficiente para pagar as despesas também apresentaram uma probabilidade 
maior $\left(\mathrm{RP}=2,22-\mathrm{IC}_{95 \%} 1,76 ; 2,80\right)$ de $\mathrm{IA}$, frente àqueles que declararam ter dinheiro para pagar as despesas.

Quanto ao estado nutricional, domicílios chefiados por indivíduos obesos apresentaram uma probabilidade maior de IA ( $\left.\mathrm{RP}=1,39-\mathrm{IC}_{95 \%} 1,13 ; 1,71\right)$, comparados aos eutróficos. Finalmente, os domicílios nos quais o responsável era fumante também apresentaram probabilidade maior $\left(\mathrm{RP}=1,28-\mathrm{IC}_{95 \%} 1,05 ; 1,56\right)$ para 0 desfecho IA, em relação aos não fumantes.

\section{Discussão}

0 presente estudo encontrou elevada prevalência de IA no município de Rio Grande, superior a um terço dos domicílios. Cerca de um em cada dez desses domicílios estava em situação de IA moderada ou grave. Considerando-se que na cidade do Rio Grande, segundo dados do último censo do IBGE, existem 66.554 domicílios, ${ }^{13}$ e sabendo-se que a prevalência de IA encontrada foi de pouco mais que $35,0 \%$, estima-se que cerca de 23.427 domicílios da cidade se encontravam em situação de IA, sendo que 4.060 em situação de IA moderada e 1.864 de IA grave.

A prevalência de IA em Rio Grande foi superior à observada para o conjunto da região Sul do país, em $2013(14,9 \%)^{6}$ e 2010 (27,3\%). ${ }^{16}$ Outro estudo de base populacional e urbano, este conduzido em Pelotas, RS, município próximo a Rio Grande, observou uma prevalência de $11,0 \%\left(\mathrm{IC}_{95 \%} 9,0 ; 13,0\right)$ de IA em $2007 .{ }^{9}$

A prevalência de IA moderada ou grave de $8,9 \%$ foi também considerada alta quando comparada aos achados dos estudos citados anteriormente, sobre a região Sul: $4,4 \%{ }^{6}$ e $7,5 \%{ }^{14}$ No estudo de Pelotas, a prevalência de IA na presença de fome, que pode ser comparada a IA grave, foi de $3,0 \%{ }^{20}$

A alta prevalência de IA encontrada pode-se explicar pela crise econômica do país, causadora de demissões em massa nas áreas portuária e petrolífera do município. A instabilidade econômica de um país implica diversos transtornos à vida de seus cidadãos, como 0 aumento da preocupação ou incerteza sobre o futuro e 0 acesso aos alimentos. ${ }^{12}$ Aumento da prevalência de IA em momentos de crise foi encontrado em algumas regiões de Portugal, em estudo realizado com dados temporais de 2011 a 2013. ${ }^{12}$

As variáveis associadas à IA no Rio Grande, como por exemplo, ser do sexo feminino, ser de raça/cor da pele não branca, ter baixa escolaridade, pertencer ao tercil de bens inferior, apresentar obesidade, ser fumante e consumir álcool abusivamente, costumam ser fatores ligados a piores desfechos em saúde. Tais achados foram também encontrados em estudo realizado com dados da $\mathrm{PNAD}^{6}$ e em outros trabalhos, nacionais e internacionais, ${ }^{21-25}$ evidenciando a necessária manutenção e melhorias dos programas de transferência de renda para indivíduos em situação de vulnerabilidade social, bem como 0 enfrentamento de problemas estruturais visando aumentar 0 acesso à renda, com maior oportunidade de emprego, visto que 0 acesso adequado à renda aumenta 0 acesso aos alimentos.

Maior número de moradores no domicílio vem-se mostrando um fator relacionado à situação de IA. ${ }^{26}$ Essa revelação também foi destacada nos dados apresentados por ocasião da última $\mathrm{PNAD},{ }^{8}$ quando foi possível observar que, conforme aumentava o número de moradores por domicílio, aumentava a probabilidade de ocorrência de IA.

Com relação à faixa etária, o estudo mostrou que os domicílios chefiados pelos mais jovens tinham maior probabilidade de apresentar IA, comparados àqueles liderados por indivíduos de maior idade, a exemplo das evidências do estudo de tendência e fatores associados à prevalência de IA, citado neste relato. ${ }^{7}$ Essa associação pode ser explicada pelo fato de indivíduos de maior faixa etária, normalmente, apresentarem maior estabilidade financeira.

Verificou-se, ademais, o fato de o chefe do domicílio ser casado representar um fator de diminuição da probabilidade de IA. Acredita-se que isso ocorra porque indivíduos vivendo em comunhão costumam apresentar maior estabilidade econômica, dada a probabilidade maior de mais de um indivíduo ser economicamente ativo.

No que diz respeito à análise das variáveis comportamentais, o uso do fumo esteve associado a maior ocorrência de IA. Acredita-se que hábitos como tabagismo possam afetar os recursos financeiros das famílias e, consequentemente, aumentar a probabilidade de ocorrência de IA. Estudo desenvolvido na Coreia, no biênio 2012-2013, concluiu que ser fumante e consumir álcool em abuso estavam associados a maior ocorrência de IA. ${ }^{27}$ Da mesma forma, estudo conduzido na Califórnia, Estados Unidos, nos anos de 2011 e 2012, mostrou que a prevalência de tabagismo é maior em domicílios caracterizados por insegurança alimentar. ${ }^{28}$

Quanto ao estado nutricional, foram encontradas associações entre obesidade do chefe do domicílio e 
maior ocorrência de IA. Este fato também foi identificado em um estudo que utilizou dados da Pesquisa Nacional de Demografia e Saúde (PNDS) 2006, quando foi demonstrada a associação de IA grave à obesidade em mulheres. ${ }^{29}$ Acredita-se que, no Brasil, o processo de transição nutricional tenha diminuído o efeito deletério da IA sobre 0 acúmulo de gordura corporal.$^{29}$ Famílias de menor poder aquisitivo passaram a adquirir alimentos de elevada densidade energética, ricos em açúcares e gorduras, em razão de seu menor custo, porém dotados de menor qualidade nutricional. Logo, o processo de transição nutricional no país está relacionado a redução da desnutrição e aumento da obesidade em famílias de baixa renda. ${ }^{30}$

Como limitações da presente pesquisa, destaca-se 0 fato de as perdas terem sido maiores em domicílios dos

Tabela 2 - Razões de prevalência, bruta e ajustada, das associações entre insegurança alimentar e variáveis sociodemográficas e comportamentais ( $n=675$ ), Rio Grande, Rio Grande do Sul, 2016

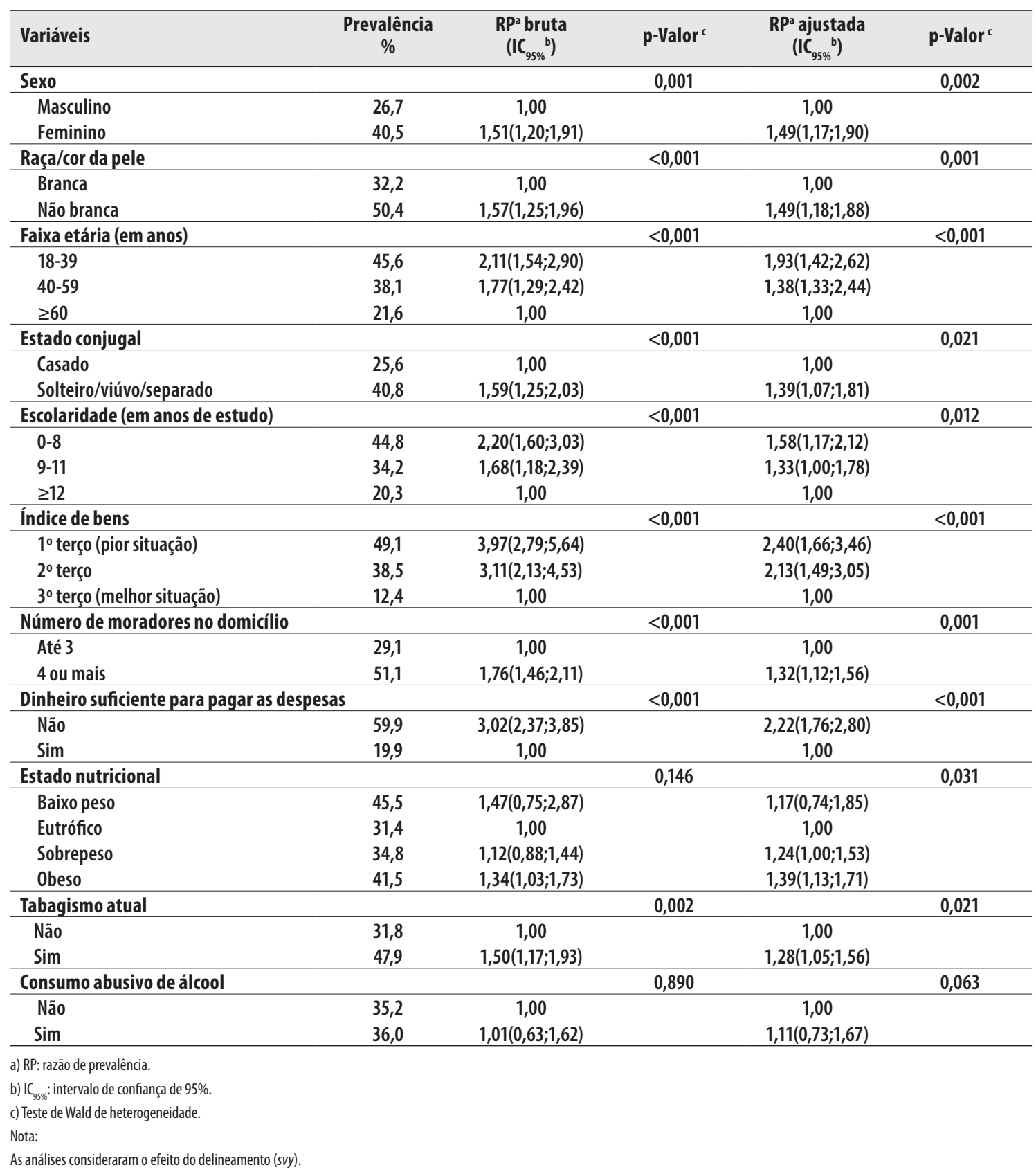


setores censitários do centro do município, e entre homens. 0 percentual geral de perdas e recusas, entretanto, revelou-se baixo. Contudo, é possível que o fato - maior perda de domicílios chefiados por indivíduos do sexo masculino - superestime a medida de sua ocorrência e impacto no estudo, visto que domicílios dos quais homens eram responsáveis obtiveram menor probabilidade de apresentar IA. Com relação à ocorrência de IA nos setores censitários da área central da cidade de Rio Grande, foi encontrada uma prevalência de 19,6\% ( IC $\left._{95 \%} 10,2 ; 32,4\right)$, menor do que a prevalência geral encontrada no estudo.

Quanto aos pontos fortes do estudo, trata-se de uma pesquisa de base populacional, o que permite investigar as condições de saúde da população e seus determinantes. Com a obtenção de dados sobre IA em Rio Grande, foi possível tanto conhecer melhor os cidadãos nessa situação como mostrar a existência de diferenças entre a prevalência de IA para a região Sul do Brasil e a prevalência encontrada no município em questão. A prevalência de IA para Rio Grande se assemelhou à estimada para a região Nordeste do país, esta com os níveis mais altos de IA. Outro ponto forte da pesquisa foi a utilização da EBIA para a mensuração do desfecho, pois essa escala, amplamente utilizada no Brasil, permite comparação com os estudos nacionais e é um instrumento validado.

A percepção da falta de dinheiro para as despesas, 0 momento econômico atual do país e especialmente de Rio Grande podem estar a agravar 0 acesso aos alimentos na quantidade e qualidade adequadas. Esses fatores associados precisam ser considerados, para a identificação

\section{Referências}

1. Bickel G, Nord M, Price C, Hamilton W, Cook J. Guide to measuring household food security, revised 2000 [Internet]. Alexandria (VA): U.S. Department of Agriculture, Food and Nutrition Service; 2000 [cited 2020 Feb 3]. 76 p. Available from: https://fns-prod. azureedge.net/sites/default/files/FSGuide.pdf

2. Conselho Nacional de Segurança Alimentar e Nutricional (BR). Documento base da III Conferência Nacional de Segurança Alimentar e Nutricional [Internet]. Brasília: Conselho Nacional de Segurança Alimentar e Nutricional; 2007 [citado 2020 fev 3] 39 p. Disponível em: http://www4.planalto.gov.br/consea/ publicacoes/site/relatorio-final-3a-conferencianacional-de-seguranca-alimentar-e-nutricional/ dos domicílios mais vulneráveis e, consequentemente, 0 manejo de políticas públicas focado nessa prioridade. A manutenção de programas de transferência de renda, redução de desemprego, aumento na renda e escolaridade dos indivíduos, além de outras ações nesse sentido, poderão minimizar a prevalência de IA.

Os resultados apresentados diferem daqueles de outras pesquisas realizadas no Sul do Brasil, revelando grande heterogeneidade entre os municípios da região, não detectada a partir de estimativas médias geradas. Portanto, é importante realizar pesquisas visando elucidar melhor o panorama de insegurança alimentar em realidades localizadas. Finalmente, cumpre salientar que os resultados do estudo foram utilizados na elaboração da política de segurança alimentar e nutricional do município de Rio Grande.

\section{Contribuição dos autores}

Dias MS contribuiu com a concepção e delineamento do estudo, análise e interpretação dos resultados e redação do manuscrito. Dumith SC contribuiu com a concepção e delineamento do estudo, revisão crítica das análises e conteúdo do manuscrito. Vaz JS contribuiu com a redação e revisão crítica do manuscrito. Susin LRO contribuiu com a concepção e delineamento do estudo e revisão crítica do manuscrito. Todos os autores aprovaram a versão final do manuscrito e são responsáveis por todos os seus aspectos, incluindo a garantia de sua precisão e integridade. documento-base-3deg-conferencia-nacional-deseguranca-alimentar-e-nutricional/view

3. Food and Agriculture Organization (FA0). 0 estado de segurança alimentar e nutricional no Brasil: um retrato multidimensional: relatório 2014 [Internet]. Brasília: Food and Agriculture Organization; 2014 [citado 2020 fev 3]. 83 p. Disponível em: https://www. mds.gov.br/webarquivos/publicacao/seguranca_ alimentar/SANnoBRasil.pdf

4. Food and Agriculture Organization (FA0). Meeting of 2015 international hunger targets: taking stock of uneven progress [Internet]. Rome: Food and Agriculture Organization; 2015 [cited 2020 Feb 3]. 56 
p. (The state of food insecurity in the world). Available from: http://www.fao.org/policy-support/resources/ resources-details/en/c/469455/

5. Victora CG, Barreto ML, Carmo Leal M, Monteiro CA, Schmidt MI, Paim J, et al. Health conditions and health-policy innovations in Brazil: the way forward. Lancet [Internet]. 2011 Jun [cited $2020 \mathrm{Feb}$ 3];377(9782):2042-53. Available from: https://www. ncbi.nlm.nih.gov/pubmed/21561659

6. Gubert MB, Benício MHDA, Santos LMP. Estimates of severe food insecurity in Brazilian municipalities. Cad Saúde Pública [Internet]. 2010 ago [citado 2020 fev 3];26(8):1595-605. Disponível em: http://www. scielo.br/pdf/csp/v26n8/13.pdf. doi: 10.1590/S0102311X2010000800013

7. Santos TG, Silveira JAC, Longo-Silva G, Ramires EKNM, Menezes RCE. Trends and factors associated with food insecurity in Brazil: the National Household Sample Survey, 2004, 2009, and 2013. Cad Saúde Pública [Internet]. 2018 [cited $2020 \mathrm{Feb} 3$ ];34(4). Available from: http://www.scielo.br/pdf/csp/v34n4/16784464-csp-34-04-e00066917.pdf. doi: 10.1590/0102$311 \times 00066917$

8. Instituto Brasileiro de Geografia e Estatística (IBGE), Ministério do Desenvolvimento Social e Combate à Fome, Ministério do Planejamento, Orçamento e Gestão. Pesquisa Nacional por Amostra de Domicílios (PNAD): segurança alimentar 2013 [Internet]. Rio de Janeiro: Instituto Brasileiro de Geografia e Estatística; 2014 [citado 2020 fev 3]. Disponível em: https:// biblioteca.ibge.gov.br/visualizacao/livros/liv91984.pdf

9. Albuquerque MFM. Food and nutritional security and the use of a human rights-based approach on the development of public policies to fight hunger and poverty. Rev Nutr [Internet]. 2009 Nov-Dec [cited 2020 Feb 3];22(6):895-903. Available from: http://www. scielo.br/pdf/rn/v22n6/v22n6a11.pdf

10. Morais DC, Dutra LV, Franceschini SCC, Priore SE. Food insecurity and anthropometric, dietary and social indicators in Brazilian studies: a systematic review. Ciênc Saúde Coletiva [Internet]. 2014 May [cited 2020 Feb 3];19(5):1475-88. Available from: http://www. scielo.br/pdf/csc/v19n5/1413-8123-csc-19-05-01475. pdf. doi: 10.1590/1413-81232014195.13012013

11. Panigassi G, Segall-Corrêa AM, Marin-León L, Pérez-Escamilla R, Sampaio M de FA, Maranha LK. Insegurança alimentar como indicador de iniqüidade: análise de inquérito populacional. Cad
Saúde Pública [Internet]. 2008 out [citado 2020 fev 3];24(10):2376-84. Disponível em: http:/www. scielo.br/pdf/csp/v24n10/18.pdf. doi: 10.1590/S0102311X2008001000018

12. Gregório MJ, Graça P, Costa A, Nogueira PJ. Time and regional perspectives of food insecurity during the economic crisis in Portugal, 20112013. Saúde Soc [Internet]. 2014 0ct-Dec [cited 2020 Feb 3];23(4):1127-41. Available from: http:// www.scielo.br/scielo.php?script=sci_arttext\&pid =S0104-12902014000401127. doi: 10.1590/S010412902014000400001

13. Instituto Brasileiro de Geografia e Estatística (IBGE). Censo demográfico 2010 resultados preliminares do universo conceitos e definições - tabelas adicionais [Internet]. Rio de Janeiro: Instituto Brasileiro de Geografia e Estatística; 2011 [citado 2020 fev 3]. Disponível em: https:/www.ibge.gov.br/estatisticas/ downloads-estatisticas.html

14. Dumith SC, Paulitsch RG, Carpena MX, Muraro MFR, Simões M0, Machado KP, et al. Planejamento e execução de um inquérito populacional de saúde por meio de consórcio de pesquisa multidisciplinar. Sci Med [Internet]. 2018 jul-set [cited 2020 Feb 3];28(3). Disponível em: https:/pesquisa.bvsalud.org/portal/ resource/pt/biblio-948784

15. Segall-Corrêa AM, Marin-León L, Melgar-Quiñonez H, Pérez-Escamilla R. Aprimoramento da escala brasileira de medida domiciliar de insegurança alimentar: recomendação de EBIA com 14 itens. Rev Nutr [Internet]. 2014 mar-abr [citado $2020 \mathrm{fev}$ 3];27(2):241-51. Disponível em: http://www.scielo. br/pdf/rn/v27n2/1415-5273-rn-27-02-00241.pdf. doi: $10.1590 / 1415-52732014000200010$

16. Facchini LA, Nunes BP, Motta JVS, Tomasi E, Silva SM, Thumé E, et al. Insegurança alimentar no Nordeste e Sul do Brasil: magnitude, fatores associados e padrões de renda per capita para redução das iniquidades. Cad Saúde Pública [Internet]. 2014 jan [citado 2020 jan 3];30(1):161-74. Disponível em: http://www.scielo.br/ pdf/csp/v30n1/0102-311X-csp-30-01-00161.pdf. doi: 10.1590/0102-311X00036013

17. Filmer D, Pritchett LH. Estimating wealth effects without expenditure data--or tears: an application to educational enrollments in states of India. Demography [Internet]. $2001 \mathrm{Feb}$ [cited $2020 \mathrm{Feb}$ 3];38(1):115-32. Available from: https://www.jstor.org/ stable/3088292?seq=1\#metadata_info_tab_contents. doi: $10.2307 / 3088292$ 
18. Marin-Leon L, Francisco PMSB, Segall-Corrêa AM, Panigassi G. Bens de consumo e insegurança alimentar: diferenças de gênero, cor de pele autorreferida e condição socioeconômica. Rev Bras Epidemiol [Internet]. 2011 set [citado 2020 fev 3];14(3):398-410. Disponível em: http://www. scielo.br/scielo.php?script=sci_arttext\&pid=\$1415790X2011000300005. doi: 10.1590/S1415790X2011000300005

19. Ministério da Saúde (BR). Secretaria de Vigilância em Saúde. Vigitel Brasil 2013: vigilância de fatores de risco e proteção para doenças crônicas por inquérito telefônico [Internet]. Brasília: Ministério da Saúde; 2014 [citado 2020 fev 3]. (Série G. Estatística e Informação em Saúde). 164 p. Disponível em: http://bvsms.saude.gov.br/ bvs/publicacoes/vigitel_brasil_2013.pdf

20. Santos JV, Gigante DP, Domingues MR. Prevalência de insegurança alimentar em Pelotas, Rio Grande do Sul, Brasil, e estado nutricional de indivíduos que vivem nessa condição. Cad Saúde Pública [Internet]. 2010 jan [citado 2020 fev 3];26(1):41-9. Disponível em: http://www.scielo.br/scielo.php?script=sci arttext\&pid=S0102-311X2010000100005. doi: 10.1590/ S0102-311X2010000100005

21. Tarasuk V, St-Germain AAF, Mitchell. Geographic and socio-demographic predictors of household food insecurity in Canada, 2011-2012. BMC Public Health [Internet]. 2019 Jan [cited $2020 \mathrm{Feb} 3$ ];19(12). Available from: https://bmcpublichealth.biomedcentral. com/articles/10.1186/s12889-018-6344-2

22. Ferreira HS, Souza MEDCA, Moura FA, Horta BL. Prevalência e fatores associados à Insegurança Alimentar e Nutricional em famílias dos municípios do norte de Alagoas, Brasil, 2010. Ciênc Saúde Coletiva [Internet]. 2014 maio [citado $2020 \mathrm{fev}$ 3];19(5):1533-42. Disponível em: http://www.scielo.br/ pdf/csc/v19n5/1413-8123-csc-19-05-01533.pdf . doi: $10.1590 / 1413-81232014195.06122013$

23. Daly A, Pollard CM, Kerr DA, Binns CW, Caraher M, Phillips M. Using cross-sectional data to identify and quantify the relative importance of factors associated with and leading to food insecurity. Int J Environ Res Public Health [Internet]. 2018 Nov [cited 2020 Feb 3];15(12):E2620. Available from: https://www. mdpi.com/1660-4601/15/12/2620. doi: 10.3390/ ijerph 15122620

24. Martin-Fernandez J, Grillo F, Parizot I, Caillavet F, Chauvin P. Prevalence and socioeconomic and geographical inequalities of household food insecurity in the Paris region, France, 2010. BMC [Internet]. 2013 Dec [cited 2020 Feb 3];13(486). Available from: https://bmcpublichealth.biomedcentral.com/ articles/10.1186/1471-2458-13-486. doi: 10.1186/14712458-13-486

25. Vuong TN, Gallegos D, Ramsey R. Household food insecurity, diet, and weight status in a disadvantaged district of Ho Chi Minh City, Vietnam: a cross-sectional study. BMC [Internet]. 2015 Mar [cited 2020 Feb 3];15:232. Available from: https://bmcpublichealth. biomedcentral.com/articles/10.1186/s12889-0151566-z. doi: 10.1186/s12889-015-1566-Z

26. Anschau F, Matsuo T, Segall-Corrêa AM. Insegurança alimentar entre beneficiários de programas de transferência de renda. Rev Nutr [Internet]. 2012 marabr [citado 2020 fev 3];25(2):177-89. Disponível em: http://www.scielo.br/scielo.php?script=sci_arttext\&p id=S1415-52732012000200001. doi: 10.1590/S141552732012000200001

27. Chung H-K, Kim 0, Kwak S, Cho Y, Lee K, Shin M-J. Household food insecurity is associated with adverse mental health indicators and lower quality of life among koreans: results from the Korea National Health and nutrition examination survey 20122013. Nutrients [Internet]. 2016 Dec [cited $2020 \mathrm{Feb}$ 3];8(12):819. Available from: https://www.ncbi.nlm. nih.gov/pmc/articles/PMC5188472/\#!po=65.5556. doi: 10.3390/nu8120819

28. Kim JE, Tsoh JY. Cigarette smoking among socioeconomically disadvantaged young adults in association with food insecurity and other factors. Prev Chronic Dis [Internet]. 2016 Jan [cited $2020 \mathrm{Feb}$ 3];13:E08. Available from: https://www.cdc.gov/pcd/ issues/2016/15_0458.htm. doi: 10.5888/pcd13.150458

29. Schlüssel MM, Silva AAM Pérez-Escamilla R, Kac G. Household food insecurity and excess weight/obesity among Brazilian women and children: a life-course approach. Cad Saúde Pública [Internet]. 2013 Feb [cited 2020 Feb 3];29(2):219-26. Available from: http:// www.scielo.br/pdf/csp/v29n2/03.pdf. doi: 10.1590/ S0102-311X2013000200003

30. Drewnowski A, Specter SE. Poverty and obesity: the role of energy density and energy costs. Am J Clin Nutr [Internet]. 2004 Jan [cited 2020 Feb 3];79(1):616. Available from: https:/academic.oup.com/ajcn/ article/79/1/6/4690070. doi: 10.1093/ajcn/79.1.6 


\section{Abstract}

Objective: to analyze the occurrence of food insecurity and associated factors in Rio Grande, Rio Grande do Sul, Brazil, in 2016. Methods: this was a crosssectional population-based study using data obtained by interviewing heads of household; Poisson regression with robust variance adjustment was used. Results: 675 households were included; food insecurity prevalence was $35.2 \%$ (95\%CI 31.6;39.0) and its occurrence was associated with heads of household being female ( $P R=1.49$ - 95\%CI1.17;1.90), not being white-skinned ( $P R=1.49$ 95\%CI1.18;1.88), being younger, unmarried ( $P R=1.39$ - 95\%CI 1.07;1.81), belonging to the lowest education bracket (PR=1.58; 95\%CI1.17;2,12), belonging to the first and second assets index tertiles, having insufficient money to meet expenses ( $P R=2.22-95 \% C I 1.76 ; 2.80)$, being obese (PR=1.39 - 95\%CI1.13;1.71), and being a smoker (PR=1.28 - 95\%CI1.05;1.56). Conclusion: food insecurity was associated with all factors studied except alcohol abuse.

Keywords: Food and Nutrition Security; Socioeconomic Factors; Social Vulnerability; Health Surveys; CrossSectional Studies.

\section{Resumen}

Objetivo: analizar la ocurrencia de inseguridad alimentaria y factores asociados en Rio Grande, Rio Grande do Sul, Brasil, en 2016. Métodos: un estudio transversal de base poblacional obtuvo información mediante la aplicación de un cuestionario a los responsables por las residencias; se utilizó la regresión de Poisson, con ajuste robusto de la varianza. Resultados: se evaluaron 675 bogares, la inseguridad alimentaria fue del $35,2 \%$ (IC $\left.{ }_{95 \%} 31,6 ; 39,0\right)$ y se asoció con que el responsable por la casa fuera del sexo femenino $\left(R P=1,49-I C_{95 \%} 1,17 ; 1,90\right)$, de raza/color de piel no blanca $\left(R P=1,49-I C_{95 \%} 1,18 ; 1,88\right)$, ser del grupo de edad más joven, no ser casada ( $\left.R P=1,39-I C_{95 \%}, 1,07 ; 1,81\right)$, pertenecer al grupo de menor escolaridad ( $R P=1,58$ - IC $\left.{ }_{95 \%} 1,17 ; 2,12\right)$, estar en el primero o segundo tercil de indice de bienes, no tener dinero suficiente para los gastos de la casa ( $\left.R P=2,22-I C_{95 \%} 1,76 ; 2,80\right)$, ser obeso $\left(R P=1,39-I C_{95 \%} 1,13 ; 1,71\right)$ y ser fumador $(R P=1,28$ $I C_{95 \%}$ 1,05;1,56). Conclusión: la inseguridad alimentaria estuvo asociada a todos los factores estudiados, excepto el abuso de alcohol.

Palabras clave: Seguridad Alimentaria y Nutricional; Factores Socioeconómicos; Vulnerabilidad Social; Encuestas Epidemiologicas; Estudios Transversales.

Recebido em 09/06/2019

Aprovado em 03/01/2020 